\title{
Comment on "Locating the source field lines of Jovian decametric radio emissions" by YuMing Wang et al.
}

\author{
Laurent Lamy 1,2,3*, Baptiste Cecconi ${ }^{1,2}$, Stéphane Aicardi ${ }^{4}$, and C. K. Louis ${ }^{5}$ \\ 1'LESIA, Observatoire de Paris, Université PSL, CNRS, Sorbonne Université, Université de Paris, 5 place Jules Janssen, 92195 Meudon, France; \\ ${ }^{2}$ Station de Radioastronomie de Nançay, Observatoire de Paris, Université PSL, CNRS, Univ. Orléans, 18330 Nançay, France; \\ 32LAM, Pythéas, Aix Marseille Université, CNRS, CNES, 38 Rue Frédéric Joliot Curie, 13013 Marseille, France; \\ ${ }^{4}$ DIO, UMS2201 CNRS, Observatoire de Paris, Université PSL, 61 avenue de l'Observatoire, 75014, Paris, France; \\ ${ }^{5}$ School of Cosmic Physics, DIAS Dunsink Observatory, Dublin Institute for Advanced Studies, Dublin, Ireland
}

Citation: Lamy, L., Cecconi, B., Aicardi, S., and Louis, C. K. (2022). Comment on "Locating the source field lines of Jovian decametric radio emissions" by YuMing Wang et al.. Earth Planet. Phys., 6(1), 10-12. http://doi.org/10.26464/epp2022018

\begin{abstract}
In this comment on the article "Locating the source field lines of Jovian decametric radio emissions" by Wang YM et al., 2020, we discuss the assumptions used by the authors to compute the beaming angle of Jupiter's decametric emissions induced by the moon lo. Their method, relying on multi-point radio observations, was applied to a single event observed on $14^{\text {th }}$ March 2014 by Wind and both STEREO A/B spacecraft from $\sim 5$ to $\sim 16 \mathrm{MHz}$. They have erroneously identified the emission as a northern (lo-B type) instead of a southern one (lo-D type). We encourage the authors to update their results with the correct hemisphere of origin and to test their method on a larger sample of Jupiter-lo emissions.
\end{abstract}

Keywords: planetary magnetosphere; Jupiter; auroral radio emissions; planet-moon interaction

\section{Introduction}

In a study published in 2020, (Locating the source field lines of Jovian decametric radio emissions, Wang YM et al., 2020, hereafter W20) proposed a method based on multi-point radio observations of Jovian decametric emissions induced by lo (hereafter lo-DAM) to accurately locate the position of lo-DAM radiosources, their host magnetic field line, and the wave emission angle at the source. Adopting the formalism proposed by Hess et al. (2008), replicated in Equation (2) of W20, the authors then converted the wave emission angle into the kinetic energy of electrons driving the radiation through the Cyclotron Maser Instability, assuming a loss cone electron distribution as its free energy source.

The proposed method is interesting and has broad implications. Differing from past studies, the authors used the up-to-date JRM09 magnetic field model computed from Juno in situ data (Connerney et al., 2018), complemented by a current sheet model still based on Voyager in situ data (Connerney et al., 1981), to minimize uncertainties in the calculated quantities.

To illustrate/validate their method, W20 applied it in their Section 3 to a single case of Io-DAM emission, observed nearly simultaneously on $14^{\text {th }}$ March 2014 by the radio instruments onboard three space probes, namely Wind/Waves (Bougeret et al., 1995) and STEREO-A and -B/Waves (Bougeret et al., 2008), from $\sim 5$ to

Correspondence to: L. Lamy, laurent.lamy@obspm.fr

Received 28 OCT 2021; Accepted 06 DEC 2021.

Accepted article online 13 JAN 2022.

(C) 2022 by Earth and Planetary Physics. $\sim 16 \mathrm{MHz}$. These observations are shown in Figure 1, replicated from Fig. 1 of W20. Assuming that the lo-DAM source region lay in the north (hence corresponding to the lo-B class of emission), W20 determined that the footprint of the magnetic field line hosting the radio emission lay ahead of the average lo UV footprint (Bonfond et al., 2009) by a $32^{\circ}$ lead angle. They derived roughly constant emission angles within $61.5^{\circ}-63.5^{\circ}$, from which they obtained electron kinetic energies varying within 12.5-18 keV. Comparing these values to those published by Zarka (1998) and Hess et al. (2008), the authors concluded that their method was thus "valid and reliable".

We disagree with the crucial assumption of W20 on the hemisphere of origin, which directly affects the results, and comment on other assumptions used by the authors, as discussed below.

\section{Hemisphere of Origin for the Io-DAM Emission Observed $14^{\text {th }}$ March 2014}

To proceed to the analysis of the Io-DAM arc observed on $14^{\text {th }}$ March 2014, the authors claim that the emission originates from the northern hemisphere on the basis of the following statement : "We do not have the polarization measurements of the event, but on 2014 March 14 the northern magnetic pole was tilted toward the spacecraft. Thus, we believe that the DAM emission should come from the northern hemisphere." However, several arguments can be opposed to this identification and support that the investigated Io-DAM emission is not an lo-B arc (northern westward source) but an lo-D one (southern westward source). 


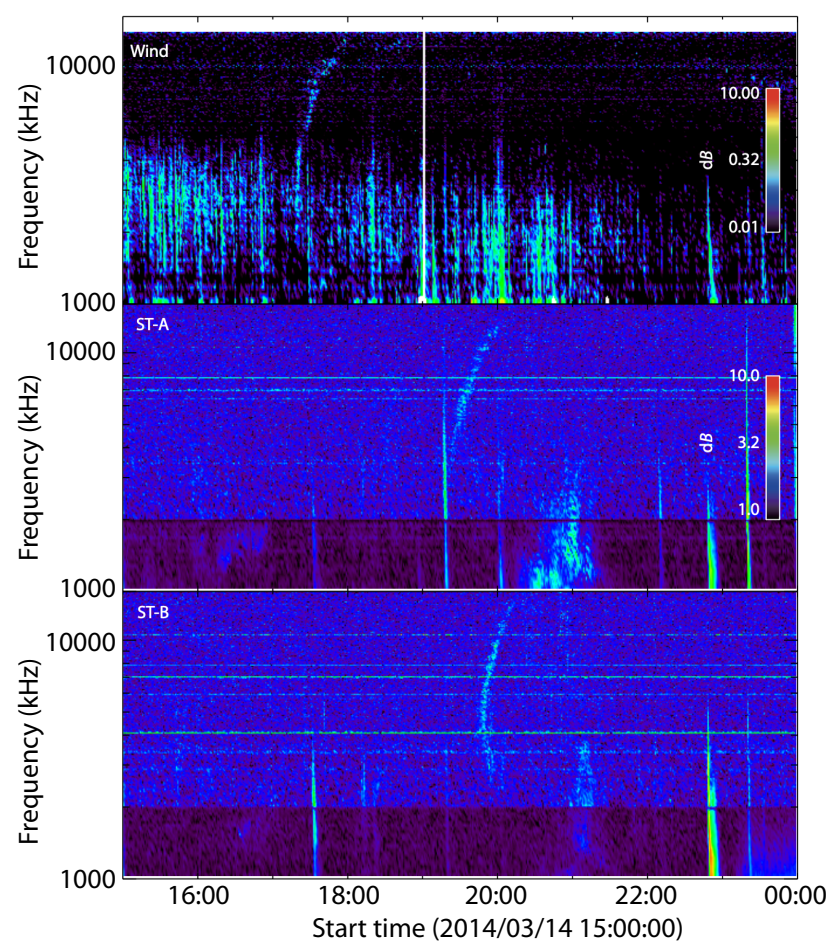

Figure 1. Io-DAM emission successively observed by Wind/Waves, and by STEREO-A and -B/Waves from $\sim 5$ to $\sim 16 \mathrm{MHz}$ (replicated from Fig. 1 of W20).

Firstly, it can be seen that the radio arc observed by Wind/Waves displays an inflexion point near $10 \mathrm{MHz}$ around 18:30 UT which is characteristic of lo-D arcs. Illustrations of the typical shape of loDAM arcs, observed over their full frequency range by Wind/Waves $(<13 \mathrm{MHz})$ and by the Nançay Decameter Array (NDA, >10 MHz), can be found in Queinnec and Zarka (1998) (see their Fig. 1 for the lo-D case).

The Jovian magnetic latitude of the Earth, applicable to the Wind spacecraft, was varying between $-8.3^{\circ}$ and $-4.1^{\circ}$ from 17:00 UT to 19:00 UT, so that southern lo-DAM sources were in more favorable view from Wind/Waves. Similarly, the arcs observed by STEREO-A and -B/Waves between 18:20 and 19:20 UT corresponded to southern magnetic latitudes varying between $-9^{\circ}$ and $-6^{\circ}$. In addition, Louis et al. (2021) recently investigated the statistical distribution of Jovian DAM emissions observed by Juno/Waves as a function of magnetic latitude and found that the northern lo-B/A arcs, which reach higher frequencies than the lo-D/C ones owing to the larger northern magnetic field amplitude, are not observed below $-5^{\circ}$ magnetic latitude (see their Fig. $3 d$ ).

The classification of Io-DAM arcs into categories can then be efficiently counter-checked from the visualization of the interval of observation on top of a classical CML-lo phase occurrence diagram, such as those built by Marques et al. (2017), Zarka et al. (2018) and references therein, from a catalogue of 26 years of NDA observations. As illustrated in Figure 2, on 14 ${ }^{\text {th }}$ March 2014, between 17:00 and 19:00 UT, Earth-based Wind/Waves observations intercepted the region of maximal probability for lo-D emissions only.

While STEREO/Waves is capable of polarization measurements (Cecconi et al., 2008), polarization measured during this event could be tracked only up to $\sim 2 \mathrm{MHz}$, below which the lo-DAM arc was not visible (as opposed to the hectometric burst observed near $\sim 21: 00$ UT). For the sake of completeness, we checked the NDA public observations of Jupiter over $10-40 \mathrm{MHz}$ that were obtained on $14^{\text {th }}$ March 2014 simultaneously to the Wind/Waves ones below $13 \mathrm{MHz}$. We could not identify any clear Jovian signature with either Right-handed or (expected) Left-handed polarization in the 10-16 MHz NDA range, where the band was unfortunately both strongly contaminated by RFIs (up to $18 \mathrm{MHz}$ ) and subject to low frequency filtering (below $14 \mathrm{MHz}$ ). Nonetheless, any sufficiently intense lo- $B$ emission should have reached frequencies >20 MHz (Queinnec and Zarka, 1998, Marques et al., 2017).
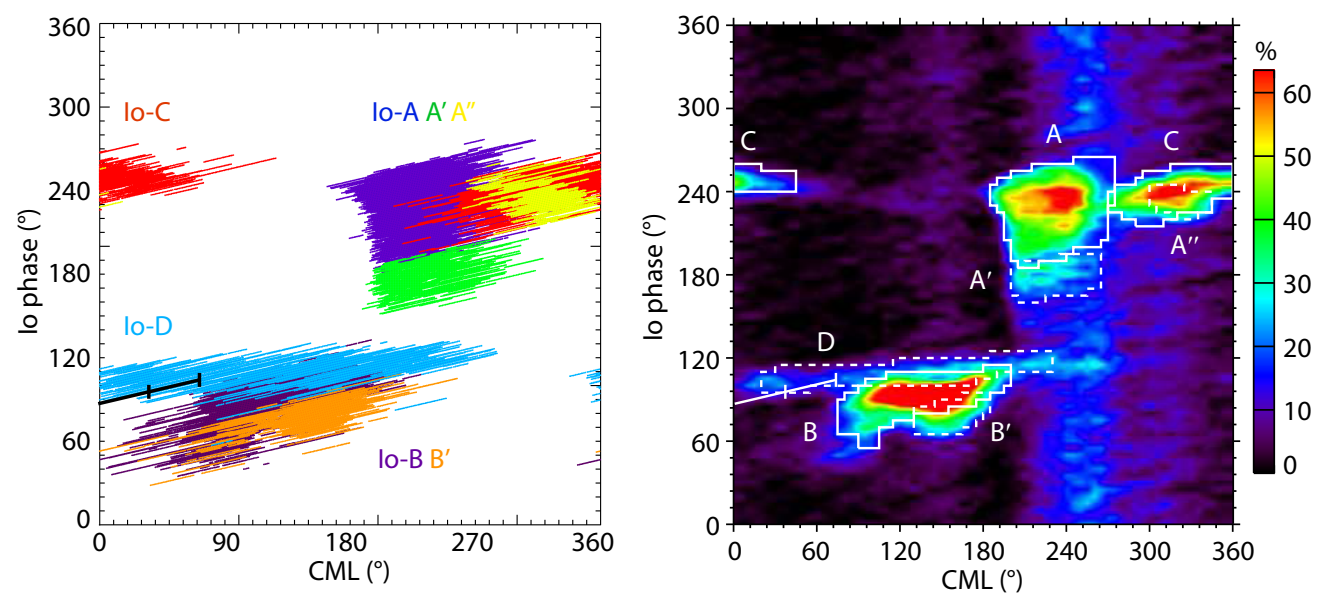

Figure 2. Plot of the Central Meridian Longitude (CML) as a function of lo phase, showing (a) the occurrence of lo-DAM arcs and (b) the occurrence probability of all Jovian DAM emissions derived from 26 years of NDA observations of Jupiter (Marques et al., 2017, Zarka et al., 2018). The solid line indicates the path of Earth-based observations on 14th March 2014 from 17:00 to 19:00 UT, when Wind/Waves recorded the lo-DAM arc displayed in Figure 1. The line precisely intercepts the lo-D region, only. Both panels were created with the online Jupiter probability tool, developed by the NDA team, at https://jupiter-probability-tool.obspm.fr 
Finally, the lead angle of $32^{\circ}$ obtained from the longitudinal difference between the footprint of the retrieved flux tube and that of the average lo UV spot is very large, and, assuming that the loDAM sources lay along the main lo UV footprint, is not consistent with the results of Bonfond et al. (2009).

\section{Other Physical Hypotheses}

To use the formalism of Hess et al. (2008), the authors define $f_{\text {ce, max }}$ (the maximal electron cyclotron frequency reached along the flux tube) as follows: "The value of $f_{\mathrm{ce}, \max }$ is set to be the value at the footprint of each field line on the 1/15.4 flattened surface of one $R_{\mathrm{J}}$, as an approximation of the frequency at the top of ionosphere." We note that the peak emission altitude of $\sim 900 \mathrm{~km}$ for the lo UV footprint (Bonfond et al., 2009) would yield a more realistic value of $f_{\text {ce,max }}$. The parametric study of lo-DAM simulations with the ExPRES code of Louis et al. (2017) has quantified the effect of the altitude on the final time-frequency shape of the arc (see their Fig. 1e).

The relevance of the formalism of Hess et al. (2008) was not at all discussed by W20; recent Juno in situ measurements of real electron distribution functions driving Jovian radio emissions provide the opportunity to assess its relevance (see e.g. Louarn et al., 2017, Louis et al., 2020).

Finally, the correspondence between the values plotted in leftand right-handed panels (which display different ranges along the $y$-axis) of Fig. 8 of W20 is not obvious, especially for the spread of dots.

\section{Conclusion}

We kindly encourage the authors to take into account the above comments to update their analysis, quantify the uncertainty on the parameters derived from assuming a wrong hemisphere of origin, and ideally extend their method to a larger set of Io-DAM emissions from both hemispheres in order to achieve more "valid and reliable" results.

\section{Acknowledgments}

We acknowledge the JPL Horizons and IMCCE/MIRIADE ephemeris services, together with Vratislav Krupar for providing STEREO/Waves high level polarization data. The Jupiter probability tool at https://jupiter-probability-tool.obspm.fr was developed by the NDA team in the frame of the MASER service supported by the Paris Astronomical Data Centre (PADC) at Observatoire de Paris. The (unshown) NDA data mentioned in the article are publicly available at https://www.obs-nancay.fr/reseau-decametrique/. We thank the CNES and CNRS/INSU programs of planetology and heliophysics.

\section{References}

Bonfond, B., Grodent, D., Gérard, J. C., Radioti, A., Dols, V., Delamere, P. A., and
Clarke, J. T. (2009). The lo UV footprint: Location, inter-spot distances and tail vertical extent. J. Geophys. Res. :Space Phys., 114(A7), A07224. https://doi.org/10.1029/2009JA014312

Bougeret, J. L., Kaiser, M. L., Kellogg, P. J., Manning, R., Goetz, K., Monson, S. J., Monge, N., Friel, L., Meetre, C. A., ... Hoang, S. (1995). WAVEs: The radio and plasma wave investigation on the wind spacecraft. Space Sci. Rev., 71(1-4), 231-263. https://doi.org/10.1007/BF00751331

Bougeret, J. L., Goetz, K., Kaiser, M. L., Bale, S. D., Kellogg, P. J., Maksimovic, M., Monge, N., Monson, S. J., Astier, P. L., .. Z Zouganelis, I. (2008). S/WAVES: The radio and plasma wave investigation on the STEREO mission. Space Sci. Rev., 136(1-4), 487-528. https://doi.org/10.1007/s11214-007-9298-8

Cecconi, B., Bonnin, X., Hoang, S., Maksimovic, M., Bale, S. D., Bougeret, J. L., Goetz, K., Lecacheux, A., Reiner, M. J., ... Zarka, P. (2008). STEREO/waves goniopolarimetry. Space Sci. Rev., 136(1-4), 549-563. https://doi.org/10.1007/s11214-007-9255-6

Connerney, J. E. P., Acuña, M. H., and Ness, N. F. (1981). Modeling the Jovian current sheet and inner magnetosphere. J. Geophys. Res. :Space Phys., 86(A10), 8370-8384. https://doi.org/10.1029/JA086iA10p08370

Connerney, J. E. P., Kotsiaros, S., Oliversen, R. J., Espley, J. R., Joergensen, J. L., Joergensen, P. S., Merayo, J. M. G., Herceg, M., Bloxham, J., . . Levin, S. M. (2018). A new model of Jupiter's magnetic field from Juno's first nine orbits. Geophys. Res. Lett., 45(6), 2590-2596. https://doi.org/10.1002/2018GL077312

Hess, S., Cecconi, B., and Zarka, P. (2008). Modeling of lo-Jupiter decameter arcs, emission beaming and energy source. Geophys. Res. Lett., 35(13), L13107. https://doi.org/10.1029/2008GL033656

Louarn, P., Allegrini, F., McComas, D. J., Valek, P. W., Kurth, W. S., André, N., Bagenal, F., Bolton, S., Connerney, J., ... Zink, J. L. (2017). Generation of the Jovian hectometric radiation: First lessons from Juno. Geophys. Res. Lett., 44(10), 4439-4446. https://doi.org/10.1002/2017GL072923

Louis, C., Louarn, P., Allegrini, F., Kurth, W. S., and Szalay, J. R. (2020). lo and Ganymede-induced decametric emission: in-situ measurements by Juno. In AGU fall meeting abstracts (Vol. 2020, SM049-08).

Louis, C. K. , Lamy, L. , Zarka, P. , Cecconi, B. , Hess, S. L. G. , Bonnin, X. (2017). Simulating Jupiter-satellite decametric emissions with ExPRES: a parametric study. In G. Fischer, et al. (Eds. ), Planetary Radio Emissions VIII (pp. 59-72). Vienna: Austrian Academy of Sciences Press.

Louis, C. K., Zarka, P., Dabidin, K., Lampson, P. A., Magalhães, F. P., Boudouma, A., Marques, M. S., Cecconi, B. (2021). Latitudinal beaming of Jupiter's radio emissions from Juno/Waves flux density measurements. J. Geophys. Res. :Space Phys., 126(10), e2021JA029435. https://doi.org/10.1029/2021JA029435

Marques, M. S., Zarka, P., Echer, E., Ryabov, V. B., Alves, M. V., Denis, L., and Coffre, A. (2017). Statistical analysis of $26 \mathrm{yr}$ of observations of decametric radio emissions from Jupiter. Astron. Astrophys., 604, A17. https://doi.org/10.1051/0004-6361/201630025

Queinnec, J., and Zarka, P. (1998). lo-controlled decameter arcs and lo-Jupiter interaction. J. Geophys. Res. :Space Phys., 103(A11), 26649-26666. https://doi.org/10.1029/98JA02435

Wang, Y. M., Jia, X. Z., Wang, C. B., Wang, S., and Krupar, V. (2020). Locating the source field lines of Jovian decametric radio emissions. Earth Planet. Phys. 4(2), 95-104. https://doi.org/10.26464/epp2020015

Zarka, P. (1998). Auroral radio emissions at the outer planets: Observations and theories. J. Geophys. Res. :Planets, 103(E9), 20159-20194. https://doi.org/10.1029/98JE01323

Zarka, P., Marques, M. S., Louis, C., Ryabov, V. B., Lamy, L., Echer, E., and Cecconi, B. (2018). Jupiter radio emission induced by Ganymede and consequences for the radio detection of exoplanets. Astron. Astropnhys., 618, A84. https://doi.org/10.1051/0004-6361/201833586 